\title{
Evaluation of LD decay and various LD-decay estimators in simulated and SNP-array data of tetraploid potato
}

\author{
Peter G. Vos ${ }^{1}$ M. João Paulo ${ }^{2}$ Roeland E. Voorrips ${ }^{1} \cdot$ Richard G. F. Visser $^{1}$ • \\ Herman J. van Eck ${ }^{1} \cdot$ Fred A. van Eeuwijk ${ }^{2}$
}

Received: 13 May 2016 / Accepted: 26 September 2016 / Published online: 3 October 2016

(C) The Author(s) 2016. This article is published with open access at Springerlink.com

\begin{abstract}
Key message The number of SNPs required for QTL discovery is justified by the distance at which linkage disequilibrium has decayed. Simulations and real potato SNP data showed how to estimate and interpret LD decay.

Abstract The magnitude of linkage disequilibrium (LD) and its decay with genetic distance determine the resolution of association mapping, and are useful for assessing the desired numbers of SNPs on arrays. To study LD and LD decay in tetraploid potato, we simulated autotetraploid genotypes and used it to explore the dependence on: (1) the number of haplotypes in the population (the amount of genetic variation) and (2) the percentage of haplotype specific SNPs (hs-SNPs). Several estimators for short-range LD were explored, such as the average $r^{2}$, median $r^{2}$, and other percentiles of $r^{2}(80,90$, and $95 \%)$. For LD decay, we looked at $\mathrm{LD}_{1 / 2,99}$, the distance at which the short-range LD is halved when using the $90 \%$ percentile of $r^{2}$ at short range, as estimator for LD. Simulations showed that the performance of various estimators for LD decay strongly depended on the number of haplotypes, although the real
\end{abstract}

Communicated by C. A. Hackett.

Electronic supplementary material The online version of this article (doi:10.1007/s00122-016-2798-8) contains supplementary material, which is available to authorized users.

Herman J. van Eck

herman.vaneck@wur.nl

1 Plant Breeding, Wageningen University and Research, P.O. Box 386, 6700 AJ Wageningen, The Netherlands

2 Biometris, Wageningen University and Research, P.O. Box 16, 6700 AA Wageningen, The Netherlands value of LD decay was not influenced very much by this number. The estimator $\mathrm{LD}_{1 / 2,90}$ was chosen to evaluate $\mathrm{LD}$ decay in 537 tetraploid varieties. $\mathrm{LD}_{1 / 2,90}$ values were $1.5 \mathrm{Mb}$ for varieties released before 1945 and $0.6 \mathrm{Mb}$ in varieties released after 2005 . $\mathrm{LD}_{1 / 2,90}$ values within three different subpopulations ranged from 0.7 to $0.9 \mathrm{Mb}$. $\mathrm{LD}_{1 / 2,90}$ was $2.5 \mathrm{Mb}$ for introgressed regions, indicating large haplotype blocks. In pericentromeric heterochromatin, LD decay was negligible. This study demonstrates that several related factors influencing LD decay could be disentangled, that no universal approach can be suggested, and that the estimation of LD decay has to be performed with great care and knowledge of the sampled material.

\section{Introduction}

Linkage disequilibrium (LD) is the non-random association between alleles at different loci in a breeding population, and can be estimated using the correlation between (SNP) markers when the SNP alleles at those loci are given numerical values, for example, 0 and 1 for bi-allelic SNPs. The amount of LD between loci is important for the success of forward genetic studies, such as Genome-Wide Association Studies (GWAS), because the extent of LD determines the required number of SNP markers and the mapping resolution (Flint-Garcia et al. 2003). Association studies originated from human genetics (Hirschhorn and Daly 2005), where large designed bi-parental populations, such as those used in plants, are impossible. The power of LD-mapping was soon recognized by plant geneticists, and therewith, extensive studies on LD were conducted in Arabidopsis thaliana (Nordborg et al. 2002; Kim et al. 2007) and in maize (Yan et al. 2009; Van Inghelandt et al. 2011). 
In the classical population genetic sense, the decay with time of LD at generation $t\left(D_{t}\right)$ is influenced by recombination frequency $(\theta)$ between two loci and the number of generations of recombination $(t)$, since the reference generation $t=0$ according to the formula $D_{t}=D_{0}(1-\theta)^{t}$. The commonly recognized factors in population genetics, such as non-random mating, selection, mutation, migration or admixture, genetic drift, or a small effective population size, will all affect estimates of LD and LD decay (Flint-Garcia et al. 2003). In heterozygous outbreeders, such as potato, pairs of SNP alleles located on the same haplotype (linked in coupling phase) can display high values of LD and subsequent LD decay is a function of the recombination frequency and the number of generations as described above. In contrast, LD between SNP alleles on different haplotypes (linked in repulsion phase) is not easily detected. For this situation, LD is hard to define and LD falls off to a very low level due to independent segregation of those haplotypes.

Self-fertilizing plants usually show less decay of LD, because in a homozygous genetic background, the recombination events are ineffective to cause LD decay. Accordingly, LD is reported to decay at short distance (100$1500 \mathrm{bp}$ ) in an outcrossing crop species, such as maize (Remington et al. 2001; Tenaillon et al. 2001), and at large distance (up to $20 \mathrm{cM}$ ) in several selfing crop species, such as barley (Kraakman et al. 2004) or durum wheat (Maccaferri et al. 2005). This is in contrast with natural populations of selfing species, such as Arabidopsis thaliana (Nordborg et al. 2002) and Medicago truncatula (Branca et al. 2011), where LD estimation suggests a much faster decay of LD (within $10 \mathrm{~kb}$ for both species). Perennial or vegetatively propagated species, such as potato and sugarcane, have a long breeding cycle and, therefore, show a limited number of historical recombination events. Hence, LD decays relatively slow (Raboin et al. 2008; D'hoop et al. 2010) in spite of the outcrossing nature of these crops.

Various approaches exist to estimate LD and LD decay. For LD, most approaches are based on the correlation calculated between marker pairs after giving numerical values to allele states, where $r^{2}$ or $D^{\prime}$ is commonly used. LD measures at short range are commonly used in an attempt to robustify LD estimates. Short-range LD is calculated across a certain interval of genetic distances between marker pairs, and then, the mean LD or a percentile of the LD can be used to define the short-range LD. Given a definition for LD, again, various methods can be used to estimate LD decay. Trend lines can be fitted based on LD measures as a function of genetic distance between markers, and this can be done for the mean LD (Yan et al. 2009), the median LD (Myles et al. 2010), or an LD percentile (Adetunji et al. 2014). In addition, the mathematical function for the trend line to describe LD decay can differ. A non-linear regression is most common (Delourme et al. 2013; Stich et al. 2013), but also more flexible functions as the LOESS function (Esteras et al. 2013) and a spline function (Zegeye et al. 2014) have been used. As an alternative to trend lines, thresholds can be defined at which $\mathrm{LD}$ stops to exist and we reach equilibrium, i.e., no effective correlation between alleles at different markers. The most commonly used threshold is $r^{2}$ of 0.1 , but a threshold of $r^{2}=0.2$ has been used as well (Delourme et al. 2013; Li et al. 2014). Adetunji et al. (2014) and Van Inghelandt et al. (2011) used a threshold based on background LD using the correlation between markers from different chromosomes. A further possibility to define an LD decay measure is to identify the distance at which half of the maximum (short range) LD has decayed (Kim et al. 2007; Lam et al. 2010; Branca et al. 2011; Zhao et al. 2011). This value will be referred to as $\mathrm{LD}_{1 / 2,90}$ in this study and describes the initial slope of the LD-decay curve. Combinations of trend line functions and LD thresholds as described above result in differences in LD-decay estimates, which may severely hinder comparison between studies and species.

The gene pool of potato offers a unique opportunity to unravel the influence of various population genetic factors that affect genome-wide decay of LD. Many varieties have been kept alive by vegetative propagation, and our panel of 537 varieties includes both ancient and modern varieties. In addition, a comprehensive pedigree database (Van Berloo et al. 2007) is available to know the number of generations between varieties of a finite gene pool comprising a few thousand varieties developed over at least two centuries. We used 14,530 SNPs of which we know the physical and genetical positions (Vos et al. 2015), and because every SNP is dated by the year of market release of the variety first showing the SNP variant, we can distinguish the recently introgressed haplotypes from the preceding ones.

In this study, several estimators for LD decay have been explored. To assist our evaluation of these estimators, we also used simulated data, varying in the number of haplotypes (i.e., the amount of genetic variation) and the percentage of haplotype specific SNPs (hs-SNPs). The performance of different LD estimators was compared (average, median, and 80, 90, and $95 \%$ percentiles for the short-range LD), as well as the performance for estimators of LD decay. Special attention was given to estimators for the distance at which half of the short-range LD decayed $\left(D_{1 / 2}\right)$. Subsequently, the short-range LD and LD decay were evaluated in a panel of 537 tetraploid varieties, genotyped with a 20K SNP array (Vos et al. 2015). Within this set of genotypes, LD decay was estimated (1) in varieties of different ages to study LD decay over time, (2) within three subpopulations to study the effect of population structure, and (3) using SNPs that have their origin in introgression breeding (admixture). Furthermore, LD decay was 
estimated using (4) SNPs with different MAF (minor allele frequencies) thresholds and (5) SNPs having different chromosomal positions (in pericentromeric heterochromatin and in euchromatin).

\section{Materials and methods}

\section{LD-decay estimators and LD-decay estimation}

Pearson $r^{2}$ formed the basis for LD estimation. The correlations were calculated on SNP dosage (0-1-2-3-4), both for simulated SNP data as well as for the SNP-array data from the variety panel. The short-range LD was calculated based on markers pairs within $100 \mathrm{~kb}$ for the variety panel and within $1 \mathrm{cM}$ for the simulated data. For the shortrange LD, five different estimators were used: (1) average of the correlation within the window; (2) $50 \%$ percentile (median); (3) $80 \%$ percentile; (4) $90 \%$ percentile; and (5) $95 \%$ percentile. For both simulated data and real data, all chromosomes were pooled to get a genome-wide LD-decay estimation.

LD decay was estimated using a spline that was fitted on a chosen short-range LD percentile using the RQSMOOTH procedure in GenStat. From the fitted spline, the distance at which half of the short-range LD had decayed was calculated. Typically, the $90 \%$ percentile of the short-range $\mathrm{LD}$ was used, $\mathrm{LD}_{1 / 2,90}$. Background $\mathrm{LD}$ was estimated in the varieties panel using 50 randomly chosen markers per chromosome. With these 600 markers, Pearson $r^{2}$ were calculated using all possible marker pairs from different chromosomes. The $95 \%$ percentile of all these pairwise correlations was used to estimate background LD.

\section{Simulated data}

To improve our understanding of LD decay and LD-decay estimators, we simulated a series of tetraploid variety panels using PedigreeSim (Voorrips and Maliepaard 2012). Panels were simulated to resemble the European potato gene pool, as perceived by earlier SNP genotyping studies (Uitdewilligen et al. 2013; Vos et al. 2015). It is assumed that most of the genetic variation present in the contemporary cultivated Solanum tuberosum gene pool descends from a limited number of founders, and thus represents a limited set of founder haplotypes (Love 1999). To test the effect of the number of (founder) haplotypes on LD decay, either $6,8,10$, or 12 founder haplotypes were simulated with allele frequencies ranging from 2.5 to $30 \%$, according to a geometric distribution Uitdewilligen et al. (2013) (Supplementary file 1). Four haplotypes were randomly assigned to ten tetraploid founder genotypes in generation 0. Initially, the proportion of haplotype specific SNPs
(hs-SNPs) was $100 \%$. With such a simulated data set, we can monitor all recombinations and their effect on LD decay over time. In addition, we varied the percentage of hs-SNPs from the initial $100 \%$ with four additional percentages of $75,50,25$, and $0 \%$ hs-SNPs, by randomly assigning a SNP to multiple founder haplotypes. A decreasing fraction of hs-SNPs implies an overall increase of the minor allele frequency, because the sequence variant is no longer unique for one of the haplotypes, but is shared by two or more of the $6,8,10$, or 12 haplotypes. Hence, the fraction of hs-SNPs is confounded with average MAF.

These two variables (4 different numbers of founder haplotypes and 5 different percentages of hs-SNPs) resulted in 20 simulated populations at generation 0 , each composed of 10 tetraploid individuals. The simulation of LD decay involved eight generations of random mating, with 200 tetraploid offspring genotypes per generation using the PedigreeSim software (Voorrips and Maliepaard 2012) with eight chromosomes (replicates) of $50 \mathrm{cM}$ each, with 501 SNP markers per chromosome separated by $0.1 \mathrm{cM}$ and a centromere at the $20 \mathrm{cM}$ position, and with random chromosome pairing and a probability of $10 \%$ of quadrivalent formation vs. $90 \%$ of bivalent pair formation. The genotypic scores of the eighth generation were used for calculations on LD and LD decay.

\section{LD in a panel of 537 potato varieties}

In addition to the simulated panels, we evaluated LD and LD decay in a panel of 537 tetraploid varieties and progenitor clones. This panel was genotyped with a 20K SNP array (Vos et al. 2015). These data were analysed using different subsets of marker and/or genotypes in five experiments, as described in the following. In experiments 1 and 2, we make use of subsets of the genotypes and experiments 3,4 , and 5 make use of different subsets of markers.

1. LD decay over time/generations It is known that $\mathrm{LD}$ decays over generations and distance. To study LD decay in time, the 537 genotypes were assigned to four groups according to age of market release. Group 1 contained genotypes released before $1945(n=45)$, group 2 contained genotypes released between 1945 and $1974(n=42)$, group 3 contained genotypes released between 1975 and $2004(n=195)$, and group 4 contained genotypes released after $2004(n=255)$. The age of a genotype is derived from the pedigree database (Van Berloo et al. 2007), where the year of market release was taken. For progenitor clones without market release, we added 10 years to the year the cross was made, as perceived from the first two digits in the seedling code, because in general, it takes ten years between making the cross and naming a variety. 
Table 1 Number of SNP markers available in various data sets for experiments to evaluate LD decay in a panel of 537 varieties

\begin{tabular}{|c|c|c|c|c|c|}
\hline $\begin{array}{l}\text { Experiment }^{\mathrm{a}} \\
\text { Chromosome }\end{array}$ & $\begin{array}{l}\text { Exp. } 1,2 \text { and } 4 \\
\text { MAF } \sim 2.5 \%^{\mathrm{b}}\end{array}$ & $\begin{array}{l}\text { Exp. } 3 \\
\text { New SNPs }>1945^{\mathrm{c}} \mathrm{MAF}>1 \%\end{array}$ & $\begin{array}{l}\text { Exp. } 4 \\
\text { MAF }>10 \%\end{array}$ & $\begin{array}{l}\text { Exp. } 4 \\
\text { MAF }>1 \%\end{array}$ & $\begin{array}{l}\text { Exp. } 5 \\
\text { Pericentromeric SNPs, MAF } \sim 2.5 \%\end{array}$ \\
\hline St $4.03 \operatorname{ch} 01$ & 780 & 145 & 694 & 969 & 139 \\
\hline St $4.03 \operatorname{ch} 02$ & 773 & 62 & 625 & 957 & 190 \\
\hline St $4.03 \operatorname{ch} 03$ & 489 & 52 & 445 & 644 & 111 \\
\hline St $4.03 \operatorname{ch} 04$ & 575 & 138 & 477 & 787 & 124 \\
\hline St $4.03 \operatorname{ch} 05$ & 548 & 40 & 459 & 793 & 142 \\
\hline St $4.03 \operatorname{ch} 06$ & 470 & 59 & 418 & 586 & 96 \\
\hline St $4.03 \operatorname{ch} 07$ & 550 & 46 & 466 & 639 & 117 \\
\hline St $4.03 \operatorname{ch} 08$ & 456 & 73 & 384 & 585 & 69 \\
\hline St $4.03 \operatorname{ch} 09$ & 424 & 87 & 382 & 568 & 88 \\
\hline St $4.03 \operatorname{ch} 10$ & 316 & 47 & 272 & 401 & 64 \\
\hline St $4.03 \operatorname{ch} 11$ & 435 & 61 & 388 & 592 & 125 \\
\hline St $4.03 \operatorname{ch} 12$ & 317 & 76 & 272 & 405 & 89 \\
\hline Total & 6133 & 886 & 5282 & 7926 & 1354 \\
\hline
\end{tabular}

${ }^{a}$ Experiments 1-5 are described in the text

b Each SNP marker is polymorphic in at least five individuals per age and/or structure group

c The SNP markers are only polymorphic in genotypes released after 1945

2. Population structure Population structure is one of the factors that may influence LD and likewise LDdecay estimates. For this purpose, the population structure was estimated using all markers in STRUCTURE (Pritchard et al. 2000), with an analysis of $K=3$ groups. Genotypes with a membership probability $>0.5$ in the STRUCTURE analysis for the "starch" subpopulation $(N=59)$ and the "Agria" subpopulation $(N=71)$ were analysed separately from the large "rest" group $(N=407)$ containing all other genotypes. In addition, population structure was estimated with a principal coordinate analysis with 710 independent markers evenly distributed over the potato genome. Information on the grouping of genotypes is given in Supplementary file 2. Group names are named as described before (D'hoop et al. 2008).

3. Admixture In Vos et al. (2015), pre-1945 (old) and post-1945 (new) genetic variants were distinguished. Pre-1945 SNPs represent sequence variants that are polymorphic in varieties released before 1945 . The majority of these SNPs continue to be polymorphic in more recent varieties. The new or post-1945 SNPs are monomorphic in old varieties and are, therefore, most likely the result of introgression breeding. Larger LD blocks are expected due to recent admixture with SNPs that originate from donor species. LD decay was analysed separately for pre-1945 and post-1945 SNPs. Old varieties were removed from the latter analysis, because all new SNPs are monomorphic.

4. MAF (minor allele frequency) thresholds LD decay was analysed using SNPs with a MAF of 1.0, 2.5, and
$10 \%$, where MAF $>2.5 \%$ is the default set of SNPs, also used in experiments 1 and 2. Variation in MAF thresholds compares well with variation in the number of haplotypes and hs-SNPs in the simulation study to understand the effect of the amount of genetic variation on LD-decay estimates.

5. Chromosomal position Recombination is suppressed in pericentromeric heterochromatin and should result in decreased LD decay. Markers located in the pericentromeric heterochromatin, as defined by Sharma et al. (2013), were analysed separately from markers positioned on chromosomal arms (used in experiments $1-4)$.

The physical distance between markers was extracted from the SNP coordinates on the potato reference genome V4.03 (Sharma et al. 2013). Based on (Sharma et al. 2013), we selected SNPs that were clearly located on the chromosomal arms. For experiments 1 and 2, a subset of 6133 markers was selected of which the minor SNP allele was present at least 5 times in each subgroup (age and subpopulation). The number of markers selected for each experiment on the variety panel is shown in Table 1 .

\section{Results}

\section{LD decay in simulated data}

As mentioned before, $\mathrm{LD}$ in tetraploid potato is mainly the result of physical linkage between two markers. We declare 

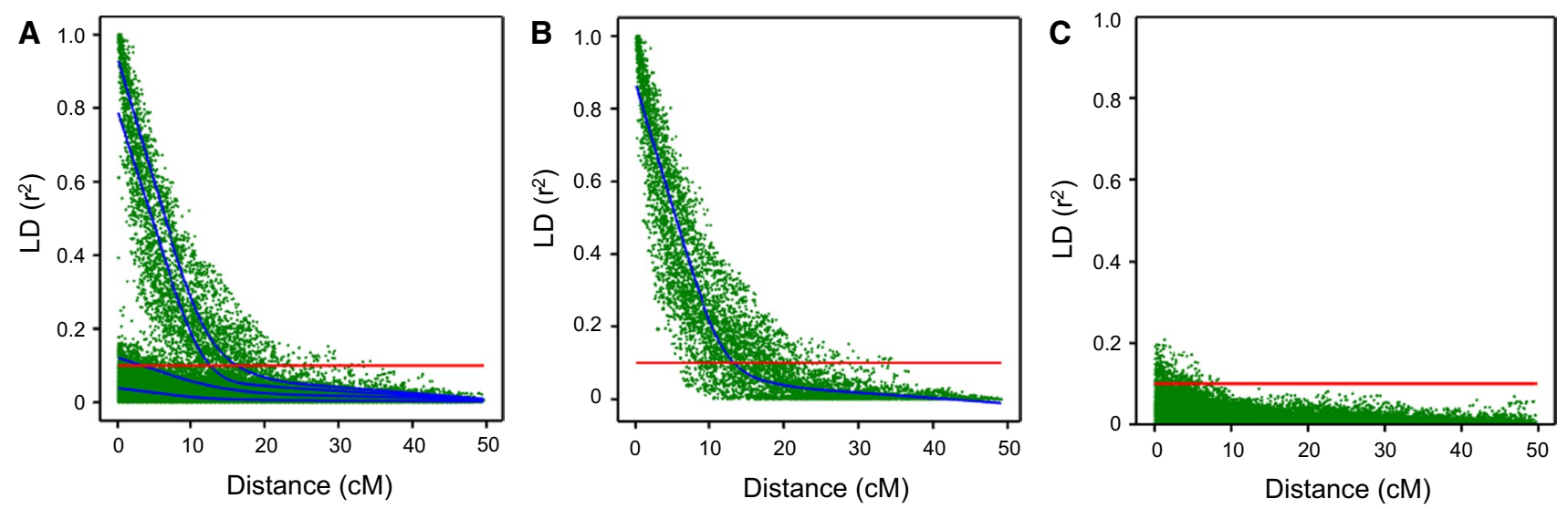

Fig. 1 LD decay (in $\mathrm{cM}$ ) in a simulated data set after eight generations of random mating starting with $100 \%$ hs-SNPs and six founder haplotypes. In a, a traditional LD-decay plot is shown. The blue lines represent splines fitted for different percentiles $(95,90,80$, and $50 \%$ ). from top to bottom. The LD threshold of 0.1 is indicated with

only the pairwise correlations that result from markers in coupling phase of interest for LD and LD-decay estimation. However, in contrast to diploids, where phasing of haplotypes is feasible (Excoffier and Slatkin 1995), phasing information for tetraploid potato is typically lacking. Consequently, LD estimation uses all pairwise allele combinations at marker pairs. This includes correlations between SNP alleles linked in coupling phase, but also less informative correlations between the SNP alleles linked in repulsion phase. To separate the informative (high LD values as a result from linkage in coupling phase) from the non-informative (low LD values as a result from linkage in repulsion phase), we have used simulated data sets with $100 \%$ haplotype specific SNPs with a known phasing of SNP alleles. Using such a data set for conventional LD-decay estimation results in an LD-decay plot, as shown in Fig. 1a. This LD-decay plot contains two kinds of pairwise correlations. Either there is a significant correlation due to the initial linkage between two hs-SNP alleles in coupling phase, or there is immediate linkage equilibrium (LE) due to random chromatid assortment of alleles linked in repulsion phase (i.e., on different haplotypes). However, the known haplotype structure of these data sets allows us to separate the informative pairwise correlation between markers linked in coupling phase from less informative correlations between markers in repulsion phase, as shown in Fig. 1b, c, respectively. The difference between gradual LD decay as a function of genetic distance and immediate linkage equilibrium due to random chromatid assortment is obvious. Figure $1 \mathrm{~b}$ shows the fitted spline drops below the threshold of $r^{2}=0.1$ at a distance of $\sim 13.5 \mathrm{cM}$. This distance of $13.5 \mathrm{cM}$ appeared to be fairly constant across simulations with $100 \%$ hs-SNPs (Fig. S1). A second important a red line. $\mathbf{b}$ Based on pairwise correlations between marker alleles from the same haplotype. Here, the blue line represents the $50 \%$ percentile. Inc, all pairwise correlations between marker alleles in linked in repulsion phase (different haplotypes) are shown (colour figure online)

observation is shown in the bottom row of Fig. S2: these graphs show that by adding more haplotypes in a simulated data set also the percentage of non-informative (generally very low) $L D$ values increases and therewith changing the estimation of LD decay using the same estimator. This second observation is in conflict with the standard formula $D_{t}=D_{0}(1-\theta)^{t}$, where the factors $t$ and $r$ suggest an independence of LD decay with the number of haplotypes in a population. Therefore, we conclude that when we aim at estimating LD and LD decay due to alleles at the same haplotype, the use of all allelic pairs causes a bias that is a function of the number of founder haplotypes, i.e., the genetic diversity.

\section{Effect of the estimator on LD-decay estimates}

In the simulated data, as an estimator for LD decay, we used the intersection of a 'significance' threshold $\left(r^{2}=0.1\right)$ and a trend line. The trend lines shown in Fig. 1a and Fig. S2 are based on spline fits on four LD percentiles (50, 80, 90, and $95 \%$ ). In addition, LD decay was calculated by the average $r^{2}$, and we looked at $\mathrm{D}_{1 / 2,90}$ (based on the $90 \%$ percentile). The LD-decay estimates differed greatly among simulated data sets and estimators (Table 2), and rarely reflected the expected value of $13.5 \mathrm{cM}$ for haplotype sharing alleles as described in the previous paragraph. Figure 1a shows that the $50 \%$ percentile (lowest blue line) is always below the threshold of $r^{2}=0.1$, and therefore here, in many other simulations, LD decay could not be determined (shown as nd in Table 2). The average $r^{2}, 50$ and $80 \%$ percentiles, always result in a major underestimation of LD decay, while using the higher percentiles (90 and $95 \%$ ) resulted in values closest to the earlier determined 
Table 2 LD-decay estimates (in cM) from simulated data

\begin{tabular}{|c|c|c|c|c|c|c|c|}
\hline \# Founder haplotypes & $\%$ hs-SNPs & Average $r^{2}$ & $50 \%$ percentile & $80 \%$ percentile & $90 \%$ percentile & $95 \%$ percentile & $\mathrm{LD}_{1 / 2,90}$ \\
\hline \multirow[t]{5}{*}{6.0} & 100 & 5.7 & nd & 4.4 & 12.8 & 16.0 & 5.3 \\
\hline & 75 & 5.5 & nd & 8.8 & 12.5 & 14.6 & 4.7 \\
\hline & 50 & 5.2 & nd & 9.7 & 12.6 & 15.0 & 3.9 \\
\hline & 25 & 4.8 & 2.0 & 9.7 & 12.3 & 14.4 & 4.7 \\
\hline & 0 & 5.1 & 3.4 & 10.2 & 12.7 & 14.7 & 4.0 \\
\hline \multirow[t]{5}{*}{8.0} & 100 & 4.2 & nd & nd & 11.5 & 14.6 & 4.9 \\
\hline & 75 & 3.2 & nd & 4.5 & 11.0 & 13.7 & 4.0 \\
\hline & 50 & 3.2 & nd & 7.3 & 11.3 & 14.1 & 4.7 \\
\hline & 25 & 2.6 & nd & 8.1 & 11.1 & 13.5 & 5.3 \\
\hline & 0 & 2.4 & 0.4 & 8.8 & 11.5 & 13.6 & 5.0 \\
\hline \multirow[t]{5}{*}{10.0} & 100 & 2.7 & nd & nd & 3.4 & 13.9 & 4.6 \\
\hline & 75 & 2.2 & nd & 0.8 & 9.2 & 13.1 & 4.1 \\
\hline & 50 & 1.7 & nd & 5.1 & 9.9 & 12.9 & 5.1 \\
\hline & 25 & 1.1 & nd & 6.6 & 10.0 & 12.2 & 5.1 \\
\hline & 0 & 1.0 & nd & 7.6 & 10.5 & 12.7 & 5.5 \\
\hline \multirow[t]{5}{*}{12.0} & 100 & 1.3 & nd & nd & nd & 12.7 & 4.3 \\
\hline & 75 & 0.6 & nd & nd & 7.1 & 12.0 & 4.1 \\
\hline & 50 & 0.2 & nd & 2.8 & 8.2 & 11.3 & 5.4 \\
\hline & 25 & 0.2 & nd & 5.0 & 9.1 & 11.8 & 5.4 \\
\hline & 0 & nd & nd & 6.1 & 9.4 & 11.6 & 5.4 \\
\hline
\end{tabular}

The values indicate the distances at which the five estimators for LD decay (average $r^{2}$ or a spline of four percentiles (50, 80, 90, and 95 \%) drop below the threshold for $r^{2}=0.1$. The values shown for $\mathrm{D}_{1 / 2}$ indicate the distances at which the $90 \%$ percentile decays to half of its initial value. Values in this table represent the average of eight replicates (chromosomes). LD estimates that never exceeded the threshold for $r^{2}=0.1$ are not defined (shown as nd). The standard errors of all estimates are very low (average $=0.25$ )

benchmark value of $13.5 \mathrm{cM}$. However, the $90 \%$ percentile also failed to estimate LD decay accurately in the data sets with 10 and 12 haplotypes. The simulations demonstrated that the number of haplotypes or the amount of genetic variation had a strong effect on LD-decay estimates. We also show that the use of the LD $90 \%$ percentile resulted in LD decay estimates closest to $13.5 \mathrm{cM}$ when six haplotypes were present and that the $95 \%$ percentile was optimal for 8 or 10 haplotypes. Table 2 suggests that even a higher percentile should be used when 12 haplotypes are present to compensate for the underestimation of LD decay.

\section{Effect of the percentage hs-SNPs on LD-decay estimates}

As explained in the previous paragraph, the LD $90 \%$ percentile suits best for the situation with six haplotypes. The five data sets using six founder haplotypes result in fairly similar LD-decay estimates ranging between 12.3 and $12.8 \mathrm{cM}$. However, the simulated data demonstrate that the different LD-decay estimates are biased in their own way by the percentage of hs-SNPs. The average $r^{2}$ as well as the $95 \%$ percentile provide estimates suggesting a slightly faster LD decay with fewer hs-SNPs. In contrast, the 50 and $80 \%$ percentiles show an opposite bias. Remarkably, the values obtained with the $\mathrm{LD}_{1 / 2,90}$ estimator do not seem to vary as much as the LD-decay values obtained with any of the other estimators. Therefore, we propose that this estimator is a very promising estimator to compare LD decay across different studies. Unfortunately, the vast majority of LD studies do not yet use $\mathrm{LD}_{1 / 2,90}$, and the outcome of $\mathrm{D}_{1 / 2}$ is difficult to compare with other LD-decay estimators.

\section{Short-range LD in simulated data sets}

We observed that $\mathrm{LD}_{1 / 2,90}$ is the most constant LD-decay estimator in the simulated data sets. The $\mathrm{LD}_{1 / 2,90}$ estimates rely on an estimation of the short-range LD. The simulations show that the short-range LD estimates (within $1 \mathrm{cM})$ are also influenced by the number of haplotypes and hs-SNPs. Two remarkable correlations are shown in Table 3. First, the average pairwise SNP correlation is halved with a doubling of the number of haplotypes. Second, the median is decreasing significantly with an increase of the percentage of hs-SNPs. Based on these trends, we can conclude that the amount of genetic variation can be approximated using the average of the shortrange $\mathrm{LD}$, and consequently, the optimal percentile can be chosen to estimate LD decay. 


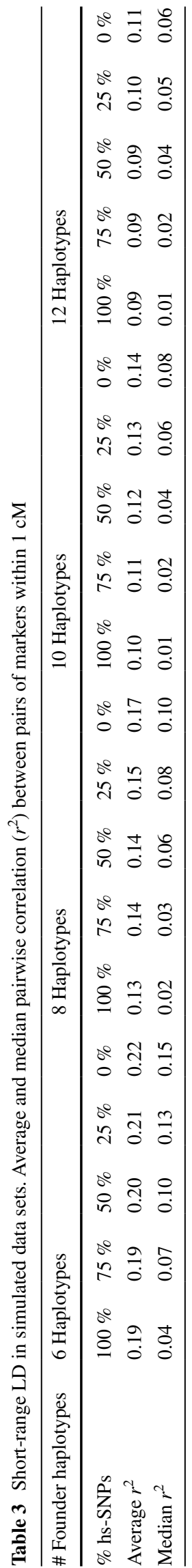

\section{Short-range $L D$ in variety panel}

Based on the empirical knowledge gained by preceding simulations, it is important to select a suitable estimator for LD analysis in real data. For this purpose, the shortrange $\mathrm{LD}$ is assessed using pairwise correlations between markers within $1 \mathrm{~kb}$, between 1 and $10 \mathrm{~kb}$, and between 10 and $100 \mathrm{~kb}$. The average $r^{2}$ and $90 \%$ percentile were the highest in the subset of pairwise correlations of markers with $10-100 \mathrm{~kb}$ distance, suggesting no LD decay within $100 \mathrm{~kb}$. Therefore, pairwise correlations of markers within $100 \mathrm{~kb}$ were used to estimate the short-range LD. These pairwise correlations were pooled over all chromosome arms. Subsequently, the average $r^{2}$ and median $r^{2}$ of the short-range LD were calculated for all experiments except experiment 5 (LD in pericentromeric heterochromatin), as shown in Table 4.

The average $r^{2}$ ranged between 0.19 and 0.22 for the different age groups and structure groups of varieties (experiments 1 and 2, respectively). For experiment 3, we compared old and new (admixed) variations. The new variation resulted in a higher average $r^{2}$ indicating fewer haplotypes and a low median $r^{2}$ suggesting a high percentage of haplotype specificity. In experiment 4 , we compared different MAF thresholds, which resulted in a lower average $r^{2}$ and median $r^{2}$ when more (lower frequent) SNPs were allowed. On average, we find values around 0.2 , which is a value similar to what we find in the simulated data sets with 6 haplotypes. In the simulated data sets, the $90 \%$ percentile performed best; therefore, we can conclude that the $90 \%$ percentile will result in a reliable estimate of LD decay in the variety panel. This $90 \%$ percentile was subsequently used to describe LD decay and to calculate the distance, where the short-range $\mathrm{LD}$ is decayed by $50 \%\left(\mathrm{LD}_{1 / 2,90}\right)$.

\section{LD decay in different age groups, experiment 1}

To evaluate how LD decays over generations within the potato genepool, the variety panel was divided into four age groups. Based on simulated data and short-range LD in the variety panel, a $90 \%$ percentile was used to describe LD decay in the four age groups (Fig. 2). LD decay in individual chromosomes did not show significant differences, and therefore, all chromosomes were pooled. The group with the oldest varieties, released before 1945, displays the most LD (black curve), whereas the group with the youngest varieties, released after 2005, displays the least LD (blue curve). Remarkably, the different age groups decay to a different background level. Therefore, the intersection between the fitted spline and the threshold of $r^{2}=0.1$ results in large differences between the age groups, which might not represent the true LD decay. Irrespective of unknown factors influencing background $\mathrm{LD}$, we observe 


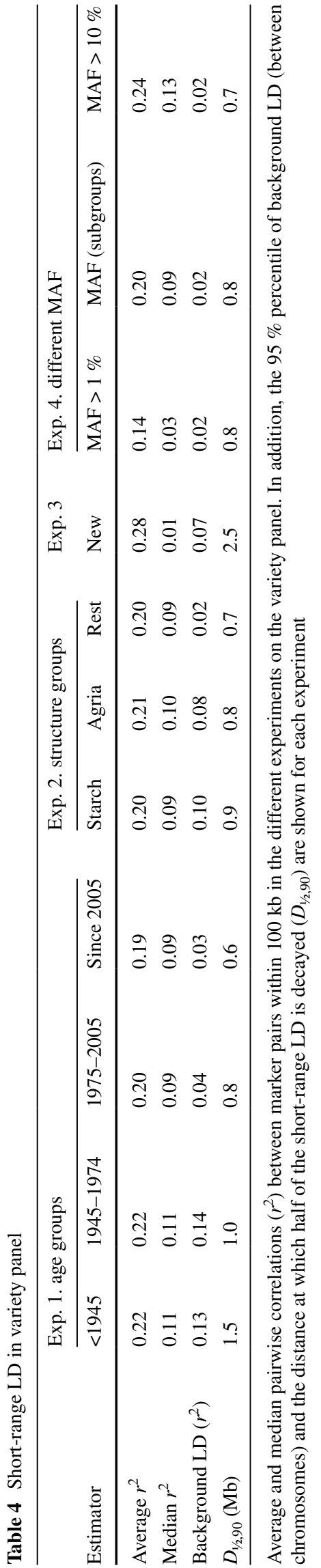

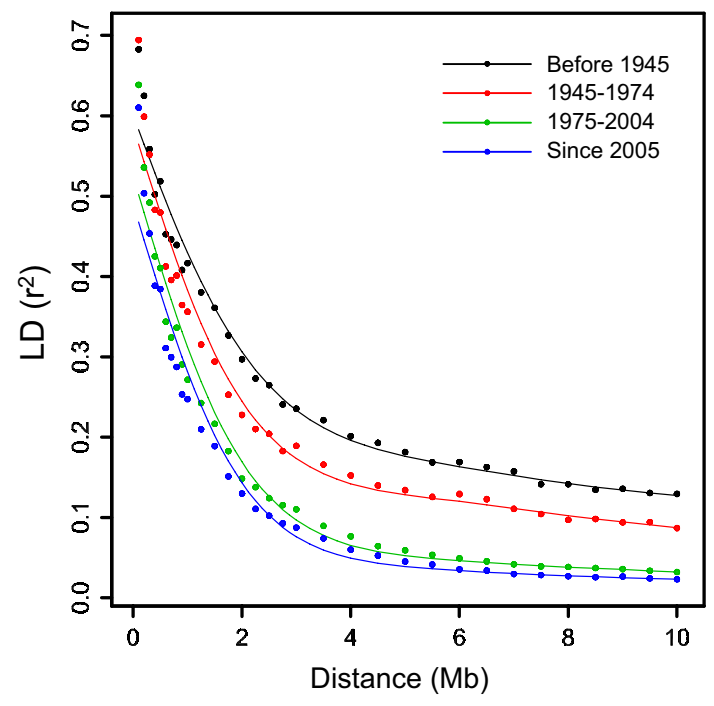

Fig. 2 LD decay over generations. The $90 \%$ percentile LD-decay splines are shown for varieties from four different age groups

that the slope of all curves flattens between a distance of 2 and $4 \mathrm{Mb}$. The $\mathrm{LD}_{1 / 2,90}$ values (Table 4 ) of the different age groups (describing the slope of the first part of the LD-decay curve) may represent the difference within the age groups better than the intersection of the spline with the threshold of $r^{2}=0.1$. The group with the older varieties reaches $\mathrm{LD}_{1 / 2,90}$ at $1.5 \mathrm{Mb}$ and the group of young varieties reaches $\mathrm{LD}_{1 / 2,90}$ at $0.6 \mathrm{Mb}$ (Table 4), suggesting that in 70 years of breeding, a substantial reduction of LD has been achieved.

\section{LD decay in different structure groups, experiment 2}

To estimate the effect of population structure on LD decay, we analysed LD decay within subgroups. Figure 3 a shows a principal coordinate (PCO) plot, where the colour of each variety represents a group membership as identified with STRUCTURE (Pritchard et al. 2000). The concordance between the analyses of PCO and STRUCTURE is high, even though the first two dimensions of the PCO explain only 3.9 and $2.8 \%$ of the variation. The red squares identify modern varieties selected for processing industry, and related to the variety Agria. The blue circles identify starch varieties and the green triangles represent all other varieties, mainly fresh consumption. In the principal coordinate and STRUCTURE analyses, additional groups have been considered. The large green group could be separated in a third group with contemporary varieties and a fourth group representing heirloom varieties. The PCO axis separating the contemporary and heirloom varieties explained less than $1 \%$ and was, therefore, not used in this experiment. Figure $3 \mathrm{~b}$ shows the spline of the $90 \%$ percentile for these 


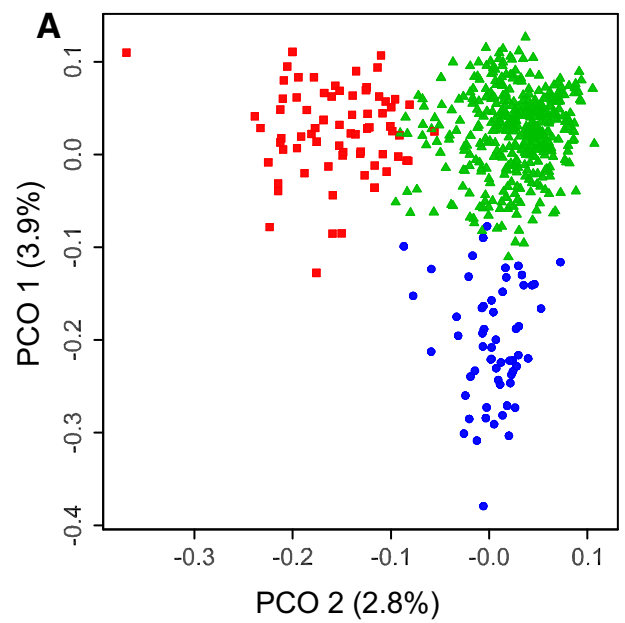

Fig. 3 LD decay and population structure. a Principal coordinate analysis showing varieties based on $>0.5$ group membership probability obtained by STRUCTURE. Green triangles represent the conventional varieties. Blue circles represent modern varieties bred for

three different subpopulations. Again, the curves flatten to different background levels between 2 and $4 \mathrm{Mb}$. However, the initial slope of these curves shows less difference compared to the age classes resulting in $\mathrm{LD}_{1 / 2,90}$ values ranging between $0.7 \mathrm{Mb}$ for the "rest" group and $0.9 \mathrm{Mb}$ for the "starch" group. Selection for specific market niches resulted in a small reduction of LD decay (Fig. 3b).

\section{The effect of admixture, MAF threshold, and chromosomal position on LD decay (experiments 3, 4, and 5)}

New sequence variants have entered the potato gene pool due to introgression breeding since 1945 (Vos et al. 2015). The consequences of admixture between wild and elite material on LD decay could be analysed by comparing LD decay among SNPs that are polymorphic in material released before or only after 1945 . Figure 4 shows the reduced LD decay perceived between SNPs on introgressed haploblocks (blue curve) that introgressed trait variation (e.g., resistance genes) could be detected with SNPs at several $\mathrm{Mb}$ distance, due to large haploblocks.

The black, turquoise, and red curves in Fig. 4 represent LD decay based on the subsets of SNPs with different minor allele frequency thresholds, where the inclusion of more infrequent SNP alleles seemingly results in faster LD decay (black curve) as compared to a more stringent threshold (MAF $>10 \%$, red curve). These curves drop below an $r^{2}$ threshold of 0.1 at significantly different physical distances. In contrast, the $\mathrm{D}_{1 / 2}$ estimates shown in Table 4 are remarkably similar and suggest a decay of $\mathrm{LD}$ at distances ranging from 0.7 to $0.8 \mathrm{Mb}$.

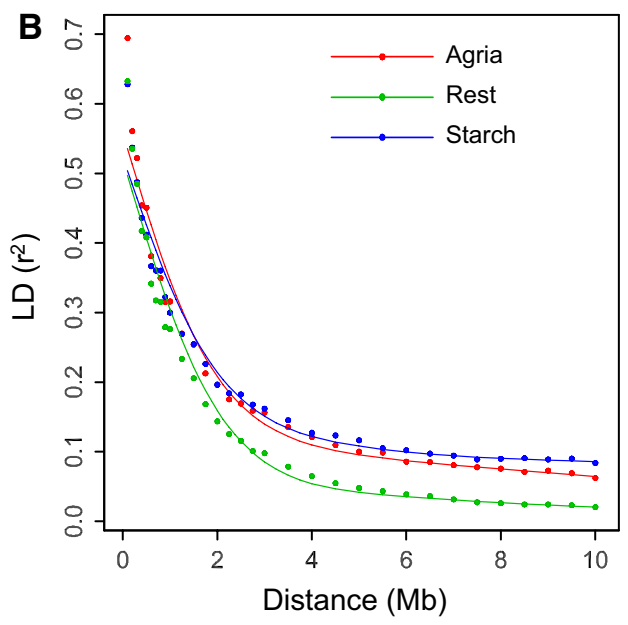

starch industry. Red squares represent modern processing varieties related to Agria. b $90 \%$ percentiles show LD decay within subgroups of varieties. Colour codes are indicated in the graph (colour figure online)

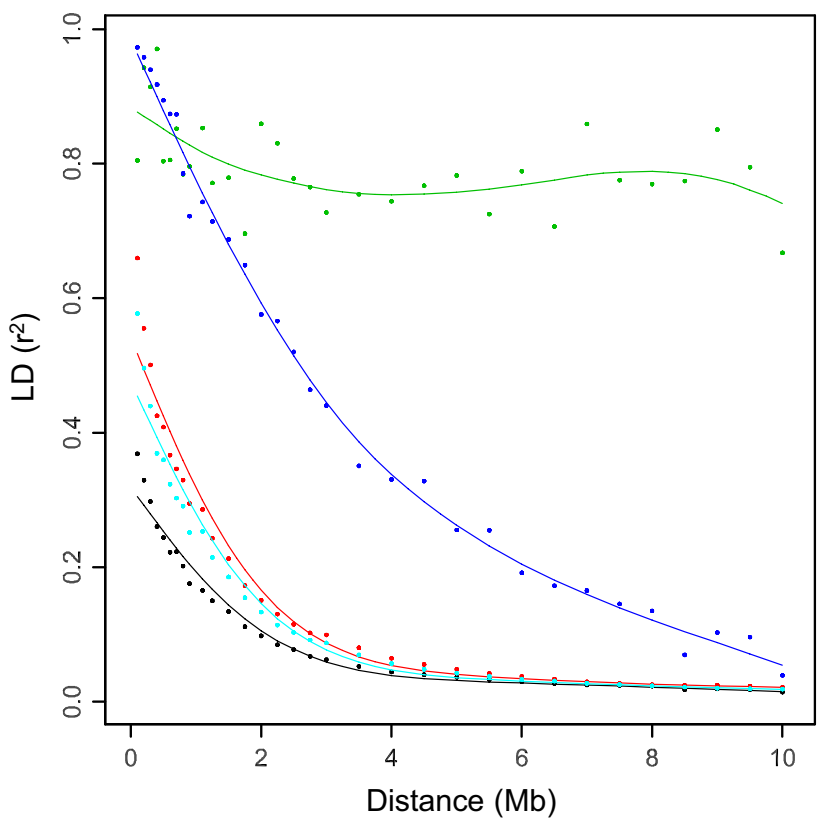

Fig. 4 LD decay perceived with different subsets of SNPs. The blue curve represents the LD decay between SNPs on haploblocks introgressed since 1945. The red, turquoise, and black curves represent LD decay between subsets of SNPs with different MAF thresholds. Red $=$ MAF $>10 \%$. Turquoise is MAF $>2.5 \%$. Black is MAF $>1 \%$. The green curve represents LD decay between SNPs with physical coordinates located in the pericentromeric heterochromatin (colour figure online)

No decay of LD up to $10 \mathrm{Mb}$ was observed between SNPs at physical coordinates that belong to the pericentromeric heterochromatin (Fig. 4 green curve) because of the suppression of recombination in centromeric regions. 


\section{Discussion}

Estimation of LD and LD decay is a highly complicated matter without obvious consensus in the literature on the preferred approach. LD decay is influenced by many factors, which usually cannot be analysed separately. In this study, we used simulated and real data representing tetraploid germplasm to understand which factors affect LD and LD-decay estimation. By doing so, we gained more insight in LD patterns in potato and its implications for GWAS and the design of genotyping arrays.

In general, the justification of the number of SNPs on the SNP array is calculated by dividing the total length of a map/genome by the distance, where a genome-wide estimate of LD reaches threshold (often $r^{2}=0.1$ ). In the results, we show that at $\sim 2 \mathrm{Mb}$, all curves start to flatten. When assuming a genome size of $400 \mathrm{Mb}$ (including the gene rich arms and excluding the pericentromeric heterochromatin (Sharma et al. 2013), we can divide this 400 by $2 \mathrm{Mb}$, suggesting that 200 markers are required per haploid genome to detect QTLs. However, one could argue that $r^{2}$ of 0.1 is too low for genome-wide QTL discovery. On the other hand, we show that within $100 \mathrm{~Kb}$, no LD decay is observed, and consequently, 4000 markers are needed to cover one haploid genome. In addition to the physical length of haploblocks, also, the number of haplotypes per locus is at stake to determine the number of SNPs on a SNP array. The number (minimum of 200 and maximum of 4000) has to be multiplied by the number of haplotypes in a population. No accurate estimates are known on the number of founder haplotypes in the potato gene pool, but when assuming an average of 10 haplotypes, then 40,000 SNPs is an upper bound for QTL discovery. The 20K SolSTW array, with 14,530 SNPs, does not reach this upper bound, but should still be able to detect sufficient QTLs.

\section{Simulated data}

The main conclusion drawn from simulated data is that estimates for genome-wide values for LD decay depend strongly on the estimator. Up to tenfold underestimations of LD decay were observed (Table 2), which has major implications for the required number of SNP markers for a GWAS, as well as the interpretation of the size of a candidate gene region. A second outcome of this study is the appreciation of the short-range LD values to gain insight in the amount of genetic diversity.

Simulations showed that the average short-range LD values halved when the amount of genetic variation was doubled because of the decreasing signal of linkage disequilibrium from marker pairs in coupling phase. This is caused by the fact that only SNPs residing on the same founder haplotype will result in high pairwise correlations. Correlation between markers on different founder haplotypes will always result in low correlation. Consequently, the percentage of high pairwise correlations will decrease with the introduction of more haplotypes.

\section{Short-range $L D$ in the variety panel}

In the literature, only relatively high averages of the shortrange $\mathrm{LD}$ values have been reported, for example, in crop species, such as wheat (Würschum et al. 2013) and maize (Yan et al. 2009), where average initial LD of $r^{2}=0.32$ and $r^{2}=0.24$, respectively, was observed, in (Wang et al. 2013) even averages of $r^{2}=0.5$ are observed. We observed a lower average $r^{2}$ for the short-range LD in our data $\left(r^{2}\right.$ is between 0.19 and 0.22 ). This suggests that in these studies, either only a limited amount of genetic variation is sampled or these studies dealt with more ascertainment bias compared to what we sampled in potato.

We propose that an estimator for LD decay should be unbiased for, or adapted to the amount of variation present in the gene pool, to allow interpretable comparisons across species. For this purpose, the average of the short-range LD within the variety panel was compared with the short-range LD in simulated data. The observed average $r^{2}$ of approximately 0.2 in the subgroups corresponds well to values observed in simulated data with six founder haplotypes. Therefore, the $90 \%$ percentile is most suitable for analysing LD decay. Indeed, this number of haplotypes is within the range of haplotypes found in the potato germplasm. Earlier haplotyping studies showed five haplotypes of the $L C Y e$ gene to 16 haplotypes of the GWD gene (Wolters et al. 2010; Uitdewilligen 2012).

Different background levels of LD makes it very difficult to determine one threshold at which linkage equilibrium is reached; therefore, we focused on the initial slope of the LD curve and used the $\mathrm{LD}_{1 / 2,90}$ values to compare LD decay within several subsets as described in experiments 1-5.

\section{LD decay in age classes}

To study how LD has decayed over the last century, we compared old and recent varieties. We observed a decrease in $\mathrm{LD}$ over the last century from a $\mathrm{D}_{1 / 2}$ of $1.5 \mathrm{Mb}$ in old varieties to $0.6 \mathrm{Mb}$ in the recent varieties, suggesting that haploblocks still have a considerable length. Long haploblocks reflect a breeding history with typically a few meiosis in a century of potato breeding, where newly introduced varieties can be as little as six meioses away from an ancestral variety from the 19th century (Van Berloo et al. 2007). In sexually reproducing crops and natural 
population, many more meioses take place annually, and therefore, one can imagine that haploblocks in potato stretches much further than in sexually propagated outbreeders. Van Inghelandt et al. (2011) also performed an analysis between old and new genotypes; however, they observe an increase in LD in more recent materials due to fixation for favourable alleles.

\section{Population structure and LD decay in structure groups}

Population structure is a confounding factor, influencing the associations in association mapping and resulting into false-positive associations. Therefore, it is essential to understand the population structure. We observed a weak population structure with $\mathrm{PCO} 1$ and $\mathrm{PCO} 2$ only explaining 3.9 and $2.8 \%$, respectively, similar to earlier potato studies (D'hoop et al. 2010; Uitdewilligen et al. 2013). Other studies (Li et al. 2010; Fischer et al. 2013; Stich et al. 2013) report on the absence of significant population structure. The difference between these studies could be explained by the sampling of the Dutch germplasm, where structure groups may result from Dutch breeding efforts. The structure group "starch" is mainly the result from specific breeding of high starch potato varieties within one breeding company. The second group is caused by the frequent use of the variety Agria as parent. Almost every variety within this group has Agria as parent or grandparent and these varieties have all been bred for the processing industry. A higher background LD was observed within these subgroups, as compared to the "rest" group, which could be the result of population structure. LD dropped below the traditional threshold of 0.1 at longer distance within these structure groups, as previously shown by (D'hoop et al. 2010). However, the $D_{1 / 2}$ values showed stable haploblock lengths, ranging from $0.7 \mathrm{Mb}$ to $0.9 \mathrm{Mb}$.

\section{Reduced decay of LD due to admixture}

Vos et al. (2015) argue that the genetic variants within the potato germplasm can be divided into groups of SNPs predating 1945 and post-1945 variation, based on the year of market introduction of the variety. In this study, LD decay was estimated using pre-1945 and post-1945 SNPs separately. The reduced decay of LD among post-1945 SNPs implies that introgressed haplotypes are substantially longer compared to haplotypes from earlier varieties. Here, we have implicitly defined the length of a haplotype as the physical size of a genomic region flanked by historical recombination events. The dating of SNPs allowed us to quantify the effect of admixture on LD decay. The data suggests that within contemporary varieties, the size of haploblocks is highly variable.

\section{Effect of MAF on LD decay}

In many studies, a restriction on the MAF is applied, where a $5 \%$ cutoff is most commonly used (Zhao et al. 2011; Delourme et al. 2013; Esteras et al. 2013; Wang et al. 2013; Würschum et al. 2013; Adetunji et al. 2014; Li et al. 2014). In some cases, a $10 \%$ (Hyten et al. 2007; Comadran et al. 2011) or even a $20 \%$ cutoff (Branca et al. 2011) is used. In this study, we showed that a restriction on the MAF significantly reduces the average $r^{2}$ and therewith influences the LD-decay estimation when the intersection of a trend line and a threshold is used (Fig. 4). The effect of MAF thresholds was previously shown by (Yan et al. 2009). However, the $D_{1 / 2}$ values (Table 4 ) were hardly affected by MAF thresholds.

\section{Final remarks}

Our analyses show that different estimators of LD and LD decay can be chosen, and this choice will result in different estimates of LD decay. In general, we conclude that the $\mathrm{LD}_{1 / 2,90}$ value offers the most consistent estimates of $\mathrm{LD}$ decay and performed best in our study. Only a few studies use this estimator (Kim et al. 2007; Lam et al. 2010; Branca et al. 2011; Zhao et al. 2011) and justify a comparative analysis across species. In potato, the distance, where half of the initial LD is decayed, is at least $600 \mathrm{~Kb}$ which is substantially longer than values observed in rice (100-300 Kb (Zhao et al. 2011) or 3-4 Kb in Arabidopsis thaliana (Kim et al. 2007) and Medicago trunctula (Branca et al. 2011). Unfortunately, no earlier study in potato used the $\mathrm{LD}_{1 / 2,90}$ estimator, preventing us to compare our data with the previous estimates of LD decay in potato. On the other hand, a general trend is that background levels of LD are reached at a distance between 2 and $4 \mathrm{Mb}$. This distance is equivalent to a genetic distance of $5-10 \mathrm{cM}$, which is in agreement with the $5 \mathrm{cM}$ reported by (D'hoop et al. 2010), and $10 \mathrm{cM}$ reported by Simko et al. (2004). The remarkable low value of LD decay in 275 bp physical distance as reported by Stich et al. (2013) can now be understood as the consequence of the choice for the an LD-decay estimator using the average $r^{2}$ in combination with a non-linear regression.

Author contribution statement Conceived and designed the experiments: PGV, HJvE, and FAvE. Performed the experiments: PGV and REV. Analysed the data: PGV, MJP, and FAvE. Wrote the manuscript: PGV and HJvE. Edited the manuscript: PGV, HJvE, FAvE, and RGFV.

Acknowledgments The collection of SNP data was financially supported by a grant from the Dutch technology foundation STW 
(Project WPB-7926). PGV is supported by a grant of Centre for BioSystems Genomics (CBSG) and by potato breeding companies Agrico Research B.V., Averis Seeds B.V., HZPC B.V., KWS POTATO B.V., and Meijer B.V.

\section{Compliance with ethical standards}

Conflict of interest The authors declare that they have no conflict of interest.

Open Access This article is distributed under the terms of the Creative Commons Attribution 4.0 International License (http://creativecommons.org/licenses/by/4.0/), which permits unrestricted use, distribution, and reproduction in any medium, provided you give appropriate credit to the original author(s) and the source, provide a link to the Creative Commons license, and indicate if changes were made.

\section{References}

Adetunji I, Willems G, Tschoep H, Bürkholz A, Barnes S, Boer M, Malosetti M, Horemans S, van Eeuwijk F (2014) Genetic diversity and linkage disequilibrium analysis in elite sugar beet breeding lines and wild beet accessions. Theor Appl Genet 127:559-571

Branca A, Paape TD, Zhou P, Briskine R, Farmer AD, Mudge J, Bharti AK, Woodward JE, May GD, Gentzbittel L, Ben C, Denny R, Sadowsky MJ, Ronfort J, Bataillon T, Young ND, Tiffin $\mathrm{P}$ (2011) Whole-genome nucleotide diversity, recombination, and linkage disequilibrium in the model legume Medicago truncatula. Proc Natl Acad Sci USA 108:E864-E870

Comadran J, Ramsay L, MacKenzie K, Hayes P, Close TJ, Muehlbauer G, Stein N, Waugh R (2011) Patterns of polymorphism and linkage disequilibrium in cultivated barley. Theor Appl Genet 122:523-531

D'hoop BB, Paulo MJ, Mank RA, Van Eck HJ, Van Eeuwijk FA (2008) Association mapping of quality traits in potato (Solanum tuberosum L.). Euphytica 161:47-60

D'hoop BB, Paulo MJ, Kowitwanich K, Sengers M, Visser RGF, van Eck HJ, van Eeuwijk FA (2010) Population structure and linkage disequilibrium unravelled in tetraploid potato. Theor Appl Genet 121:1151-1170

Delourme R, Falentin C, Fomeju BF, Boillot M, Lassalle G, André I, Duarte J, Gauthier V, Lucante N, Marty A, Pauchon M, Pichon $\mathrm{J}-\mathrm{P}$, Ribière $\mathrm{N}$, Trotoux $\mathrm{G}$, Blanchard $\mathrm{P}$, Rivière $\mathrm{N}$, Martinant $\mathrm{J}-\mathrm{P}$, Pauquet J (2013) High-density SNP-based genetic map development and linkage disequilibrium assessment in Brassica napus L. BMC Genom 14:1-18

Esteras C, Formisano G, Roig C, Díaz A, Blanca J, Garcia-Mas J, Gómez-Guillamón ML, López-Sesé AI, Lázaro A, Monforte AJ, Picó B (2013) SNP genotyping in melons: genetic variation, population structure, and linkage disequilibrium. Theor Appl Genet 126:1285-1303

Excoffier L, Slatkin M (1995) Maximum-likelihood estimation of molecular haplotype frequencies in a diploid population. Mol Biol Evol 12:921-927

Fischer M, Schreiber L, Colby T, Kuckenberg M, Tacke E, Hofferbert H-R, Schmidt J, Gebhardt C (2013) Novel candidate genes influencing natural variation in potato tuber cold sweetening identified by comparative proteomics and association mapping. BMC Plant Biol 13:1
Flint-Garcia SA, Thornsberry JM, Buckler ES IV (2003) Structure of linkage disequilibrium in plants. Annu Rev Plant Biol 54:357-374

Hirschhorn JN, Daly MJ (2005) Genome-wide association studies for common diseases and complex traits. Nat Rev Genet 6:95-108

Hyten DL, Choi I-Y, Song Q, Shoemaker RC, Nelson RL, Costa JM, Specht JE, Cregan PB (2007) Highly variable patterns of linkage disequilibrium in multiple soybean populations. Genetics 175:1937-1944

Kim S, Plagnol V, Hu TT, Toomajian C, Clark RM, Ossowski S, Ecker JR, Weigel D, Nordborg M (2007) Recombination and linkage disequilibrium in Arabidopsis thaliana. Nat Genet 39:1151-1155

Kraakman ATW, Niks RE, Van den Berg PMMM, Stam P, Van Eeuwijk FA (2004) Linkage disequilibrium mapping of yield and yield stability in modern spring barley cultivars. Genetics 168:435-446

Lam H-M, Xu X, Liu X, Chen W, Yang G, Wong F-L, Li M-W, He W, Qin N, Wang B, Li J, Jian M, Wang J, Shao G, Wang J, Sun SS-M, Zhang G (2010) Resequencing of 31 wild and cultivated soybean genomes identifies patterns of genetic diversity and selection. Nat Genet 42:1053-1059

Li L, Paulo MJ, van Eeuwijk FA, Gebhardt C (2010) Statistical epistasis between candidate gene alleles for complex tuber traits in an association mapping population of tetraploid potato. Theor Appl Genet 121:1303-1310

Li X, Han Y, Wei Y, Acharya A, Farmer AD, Ho J, Monteros MJ, Brummer EC (2014) Development of an alfalfa SNP array and its use to evaluate patterns of population structure and linkage disequilibrium. PLoS One 9:e84329

Love SL (1999) Founding clones, major contributing ancestors, and exotic progenitors of prominent North American potato cultivars. Am J Potato Res 76:263-272

Maccaferri M, Sanguineti MC, Noli E, Tuberosa R (2005) Population structure and long-range linkage disequilibrium in a durum wheat elite collection. Mol Breed 15:271-290

Myles S, Chia J-M, Hurwitz B, Simon C, Zhong GY, Buckler E, Ware D (2010) Rapid genomic characterization of the genus vitis. PloS One 5:e8219

Nordborg M, Borevitz JO, Bergelson J, Berry CC, Chory J, Hagenblad J, Kreitman M, Maloof JN, Noyes T, Oefner PJ, Stahl EA, Weigel D (2002) The extent of linkage disequilibrium in Arabidopsis thaliana. Nat Genet 30:190-193

Pritchard JK, Stephens M, Donnelly P (2000) Inference of population structure using multilocus genotype data. Genetics 155:945-959

Raboin L-M, Pauquet J, Butterfield M, D'Hont A, Glaszmann J-C (2008) Analysis of genome-wide linkage disequilibrium in the highly polyploid sugarcane. Theor Appl Genet 116:701-714

Remington DL, Thornsberry JM, Matsuoka Y, Wilson LM, Whitt SR, Doebley J, Kresovich S, Goodman MM, Buckler ES (2001) Structure of linkage disequilibrium and phenotypic associations in the maize genome. Proc Natl Acad Sci USA 98:11479-11484

Sharma SK, Bolser D, de Boer J, Sønderkær M, Amoros W, Carboni MF, D'Ambrosio JM, de la Cruz G, Di Genova A, Douches DS, Eguiluz M, Guo X, Guzman F, Hackett CA, Hamilton JP, Li G, Li Y, Lozano R, Maass A, Marshall D, Martinez D, McLean K, Mejía N, Milne L, Munive S, Nagy I, Ponce O, Ramirez M, Simon R, Thomson SJ, Torres Y, Waugh R, Zhang Z, Huang S, Visser RGF, Bachem CWB, Sagredo B, Feingold SE, Orjeda G, Veilleux RE, Bonierbale M, Jacobs JME, Milbourne D, Martin DMA, Bryan GJ (2013) Construction of reference chromosomescale pseudomolecules for potato: Integrating the potato genome with genetic and physical maps. G3 Genes Genom Genet 3:2031-2047 
Simko I, Costanzo S, Haynes KG, Christ BJ, Jones RW (2004) Linkage disequilibrium mapping of a Verticillium dahliae resistance quantitative trait locus in tetraploid potato (Solanum tuberosum) through a candidate gene approach. Theor Appl Genet 108:217-224

Stich B, Urbany C, Hoffmann P, Gebhardt C (2013) Population structure and linkage disequilibrium in diploid and tetraploid potato revealed by genome-wide high-density genotyping using the SolCAP SNP array. Plant Breed 132:718-724

Tenaillon MI, Sawkins MC, Long AD, Gaut RL, Doebley JF, Gaut BS (2001) Patterns of DNA sequence polymorphism along chromosome 1 of maize (Zea mays ssp. mays L.). Proc Natl Acad Sci USA 98:9161-9166

Uitdewilligen JGAML (2012) Discovery and genotyping of existing and induced DNA sequence variation in potato. Thesis, Wageningen University, Wageningen, NL. http://edepot.wur.nl/231154

Uitdewilligen JGAML, Wolters A-MA, Bjorn B, Borm TJA, Visser RGF, van Eck HJ (2013) A next-generation sequencing method for genotyping-by-sequencing of highly heterozygous autotetraploid potato. PLoS One 8:e62355

Van Berloo R, Hutten RCB, Van Eck HJ, Visser RGF (2007) An online potato pedigree database resource. Potato Res 50:45-57

Van Inghelandt D, Reif JC, Dhillon BS, Flament P, Melchinger AE (2011) Extent and genome-wide distribution of linkage disequilibrium in commercial maize germplasm. Theor Appl Genet 123:11-20

Voorrips RE, Maliepaard CA (2012) The simulation of meiosis in diploid and tetraploid organisms using various genetic models. BMC Bioinform 13:1

Vos PG, Uitdewilligen JGAML, Voorrips RE, Visser RGF, van Eck HJ (2015) Development and analysis of a 20K SNP array for potato
(Solanum tuberosum): an insight into the breeding history. Theor Appl Genet 128:2387-2401

Wang Y-H, Upadhyaya HD, Burrell AM, Sahraeian SME, Klein RR, Klein PE (2013) Genetic structure and linkage disequilibrium in a diverse, representative collection of the $\mathrm{C} 4$ model plant, Sorghum bicolor. G3 Genes Genom Genet 3:783-793

Wolters A-MA, Uitdewilligen JGAML, Kloosterman BA, Hutten RCB, Visser RGF, van Eck HJ (2010) Identification of alleles of carotenoid pathway genes important for zeaxanthin accumulation in potato tubers. Plant Mol Biol 73:659-671

Würschum T, Langer SM, Longin CFH, Korzun V, Akhunov E, Ebmeyer E, Schachschneider R, Schacht J, Kazman E, Reif JC (2013) Population structure, genetic diversity and linkage disequilibrium in elite winter wheat assessed with SNP and SSR markers. Theor Appl Genet 126:1477-1486

Yan J, Shah T, Warburton ML, Buckler ES, McMullen MD, Crouch J (2009) Genetic characterization and linkage disequilibrium estimation of a global maize collection using SNP markers. PloS One 4:e8451

Zegeye H, Rasheed A, Makdis F, Badebo A, Ogbonnaya FC (2014) Genome-wide association mapping for seedling and adult plant resistance to stripe rust in synthetic hexaploid wheat. PloS One 9:e105593

Zhao K, Tung C-W, Eizenga GC, Wright MH, Ali ML, Price AH, Norton GJ, Islam MR, Reynolds A, Mezey J, McClung AM, Bustamante CD, McCouch SR (2011) Genome-wide association mapping reveals a rich genetic architecture of complex traits in Oryza sativa. Nat Commun 2:467 